\title{
ADHD genetic liability and physical health outcomes - A two-sample Mendelian randomization study
}

Beate Leppert, Ph.D. ${ }^{1,2}$, Lucy Riglin, Ph.D. ${ }^{3}$, Christina Dardani, MSc. ${ }^{4}$, Ajay Thapar, Ph.D. ${ }^{3}$, James R Staley, Ph.D. ${ }^{1,2}$, Kate Tilling, Prof. ${ }^{1,2}$, George Davey Smith, Prof. ${ }^{1,2}$, Anita Thapar, Prof. ${ }^{3}$, Evie Stergiakouli, Ph.D. ${ }^{1,2,5}$

${ }^{1}$ MRC Integrative Epidemiology Unit, University of Bristol, Bristol, UK

${ }^{2}$ Population Health Sciences, Bristol Medical School, University of Bristol, Bristol, UK

${ }^{3}$ MRC Centre for Neuropsychiatric Genetics and Genomics, Cardiff University, Cardiff, UK

${ }^{4}$ Centre of Academic Mental Health, Population Health Sciences, Bristol Medical School, University of Bristol, Bristol, UK

${ }^{5}$ School of Oral and Dental Sciences, University of Bristol, Bristol, UK

\section{Corresponding author:}

Dr. Beate Leppert

MRC Integrated Epidemiology Unit, University of Bristol, Oakfield House, Oakfield Grove, Bristol BS8 2BN, UK

E-mail: beate.leppert@bristol.ac.uk

Phone: +441173310192

Keywords: ADHD, two-sample Mendelian Randomization, Physical health

Word count: 3000 


\section{Abstract}

Objective: Attention-deficit/hyperactivity disorder (ADHD) has been associated with a broad range of physical health problems, including cardiometabolic, neurological and immunological conditions. Determining whether ADHD plays a causal role in these associations is of great importance for treatment and prevention but also because comorbid health problems further increase the serious social and economic impacts of ADHD on individuals and their families.

Methods: We used a two-sample Mendelian randomization (MR) approach to examine the causal relationships between genetic liability for ADHD and previously implicated physical health conditions. 11 genetic variants associated with ADHD were obtained from the latest summary statistics. Consistent effects obtained from IVW, weighted median and MR Egger methods were taken forward for sensitivity analysis, including bidirectional MR and multivariable MR (MVMR).

Results: We found evidence of a causal effect of genetic liability for ADHD on childhood obesity (OR:1.29 (95\% CI:1.02,1.63)) and coronary artery disease (CAD) (OR:1.11 (95\% CI:1.03,1.19)) with consistent results across different MR approaches. There was further evidence for a bidirectional relationship between genetic liability for ADHD and childhood obesity. The effect of genetic liability for ADHD on CAD was independent of smoking heaviness but was attenuated when simultaneously controlling for childhood obesity. There was little evidence for a causal effect on other cardiometabolic, immunological, neurological disorders and lung cancer.

Conclusion: Our findings strengthen the argument for early treatment and support for children with ADHD and their families and especially promoting physical activity and providing them with dietary advice to reduce future risk for developing CAD. 


\section{Key Message}

- Epidemiological studies have reported observational associations between ADHD and adult onset physical health outcomes.

- Mendelian Randomization can be used to assess causal associations for ADHD on health outcomes that would traditionally require long term follow-up and may suffer confounding

- We found that genetic liability for ADHD was associated with coronary artery disease and there was evidence for a bidirectional association between genetic liability for ADHD and childhood obesity

- Multivariable mendelian randomization suggests that the link between genetic liability and coronary artery disease might partially act through childhood obesity but was independent of smoking heaviness

- There was little evidence of a causal of ADHD on other cardiometabolic and immunological diseases. 


\section{INTRODUCTION}

Attention-deficit/hyperactivity disorder (ADHD) is the most common neurodevelopmental condition with an onset typically in early childhood and a worldwide prevalence of about $5 \%$ in school-aged children and about $2-3 \%$ in adults $(1,2)$. In $65 \%$ of children diagnosed with ADHD symptoms impairment persist into adulthood and can be associated with psychiatric problems, social and occupational difficulties (3). ADHD is associated with higher mortality and morbidity rates(4) and a broad range of physical health problems. These include metabolic problems (including obesity and type 2 diabetes mellitus), cardiovascular problems (such as hypertension(5) and other risk factors for cardiovascular disease (CVD)), neurological problems (including epilepsy and migraine(6)), allergic/inflammatory disorders (including asthma (6-8), allergic rhinitis and coeliac disease)(6) and some types of cancer.

Determining whether ADHD causally contributes to these health problems is of great importance because comorbid health problems further increase the serious social and economic impacts of $A D H D$ on individuals and society $(9,10)$. However, there are multiple possible explanations for the association between ADHD and physical health problems:

1. ADHD could cause physical health problems by increasing risk for an unhealthy lifestyle and risk behaviours, e.g. via smoking, excess alcohol consumption, sedentary lifestyle and dietary choices all of which appear to influence physical ill-health.

2. ADHD could be associated with physical health outcomes because of shared genetic variants (pleiotropic effects).

3. Shared environmental risk (pre- and postnatal factors) may confound the association between ADHD and poor physical health.

Causal relationships between ADHD and physical health problems cannot be resolved by conventional observational studies because of issues including selection bias, reverse causation and residual confounding (11). However recent advances in genome-wide association studies enable the use of genetic variants strongly associated with ADHD (12) as 
proxies (instrumental variables) for the life-time risk of ADHD and to study causal effects of genetic liability for ADHD on physical health outcomes using Mendelian randomization (MR). The rationale behind MR is that genetic variants can be used as theoretically un-confounded proxies for that exposure since they are determined randomly at conception and segregated through to viable offspring independently of environmental influences(13, 14).

In this study, we aim to investigate causal relationships between genetic liability for ADHD and metabolic, cardiovascular, inflammatory and neurological conditions previously observed to be associated with ADHD, using a two-sample MR design. 


\section{METHODS}

\section{Two-sample MR}

MR utilizes genetic variants as proxies for an exposure of interest and allows the estimation of an un-confounded causal association between exposure and outcome, if certain assumptions(13) hold true:

1. The genetic variants are strongly associated with the exposure of interest.

2. The genetic variants are independent of confounders of exposure and outcome.

3. The genetic variants do not affect the outcome except through the exposure (exclusion restriction criterium). If they affect the outcome through other pathways, this is called horizontal pleiotropy.

Within a two-sample MR approach GWAS summary statistics are used to assess both genetic variant-exposure and genetic variant-outcome associations. All steps of the twosample MR performed here are explained in more detail below and summarized in Figure 1. Analyses were conducted using the TwoSampleMR package version 0.4 .14 for $R$ (version 3.4.1).

\section{ADHD GWAS (exposure)}

Genetic variants associated with ADHD (the exposure) we identified in the summary statistics for European ancestry participants from the combined PGC+iPSYCH metaanalysis of ADHD including 19099 cases and 34194 controls (12). Log odds ratio (OR) and standard error estimates for thirteen SNPs with $\mathrm{p}<1 \times 10^{-7}$ were extracted after clumping $\left(r^{2}=0.001\right.$ within $\left.10,000 \mathrm{~kb}\right)$ and removal of palindromic sequences (Table $S 1$ in the Supplement).

\section{GWAS on physical health problems (outcome)}

We used freely available GWAS summary statistics from predominantly European ancestry populations on physical health problems that have been previously reported as being 
associated with ADHD (6-8). The outcomes included cardiometabolic(15-19), neurological(20), immunological diseases(18, 21-24) and lung cancer (25) (detailed overview in Table 1 and Table S2), because of the strong link between ADHD and smoking behaviour. An exploratory analysis was conducted for metabolic markers derived from the 2016 GWAS from Kettunen et al., since they have been implicated as risk factors for CAD and obesity (26). GWAS derived from UK biobank by the Neale lab (http://www.nealelab.is/uk-biobank) are reported on a risk difference scale and were transformed onto the log OR scale (see supplementary material). All outcome GWAS are thought to be independent of the PGC+iPSYCH sample. The GWAS for coronary artery disease and myocardial infarction are derived from a mixed population sample with $77 \%$ white European participants.

SNPs associated with ADHD were extracted from the respective outcome GWAS (Table S3). If SNPs could not be extracted (due to differences in genotype chips, reference panels or imputation methods between studies), we searched for proxies using SNiPA with $r^{2}=0.9$ (https://snipa.helmholtz-muenchen.de/snipa3/).

\section{Assessing instrument strength}

Instrument strength (assumption (1) of MR) was assessed using the F-statistic, which is equal to the ratio of the mean square of the model estimates to the mean square of the model error for each genetic variant. If $\mathrm{F}>10$, as a rule of thumb, results should not suffer from weak instrument bias(27).

\section{Estimating causal effects using a two-sample MR approach}

The SNP-exposure and SNP-outcome associations were assessed using an inversevariance weighted approach (IVW), a weighted median approach and MR-Egger regression. All three methods require different assumptions on instrument validity to be true and should be interpreted alongside each other. IVW assumes all instruments are valid with no horizontal pleiotropy and therefore restricts the regression intercept to zero (28). Weighted median provides a causal estimate if at least $50 \%$ of instruments can be considered valid 
(29). MR-Egger relaxes the assumption that the outcome is affected only via the exposure and includes a random intercept coefficient in the weighted regression, which reduces power compared to the other two methods. The intercept coefficient is then equivalent to the overall pleiotropic effect and the slope coefficient displays the causal estimate under the assumption that the pleiotropic effects of the genetic variants on the outcome are not proportional to the variants' effects on the risk factor of interest (30).

Odds ratios (OR) for associations between binary exposures and binary outcomes in twosample MR studies are interpreted as the OR for outcome per unit increase in the log odds ratio of the exposure.

We present multiple testing uncorrected estimates (while acknowledging the number of correlated phenotypes that have been tested) and focus on consistent results across MR methods to assess the strength of evidence for a causal effect.

\section{Assessing heterogeneity}

Cochrane's $Q$ test was used to test for heterogeneity in the estimates from the different instruments in IVW. A large test statistic indicates that the estimated causal effects vary across the different instruments. An adaption of the $\mathrm{I}^{2}$ statistics often employed in metaanalysis, called $\mathrm{I}_{\mathrm{GX}}{ }$, was used to test for heterogeneity in MR Egger regression(31). Low $\mathrm{I}_{\mathrm{GX}}{ }$ values $(<90 \%)$ suggest heterogeneity and indicate potential violations of the "no measurement error assumption" (NOME) assumption in MR Egger and lead to regression dilution bias. We also performed SIMEX adjusted MR Egger regression where appropriate (R package SIMEX version 1.7) (31).

\section{Bidirectional MR}

When a potentially causal effect of genetic liability for ADHD on a health outcome was detected, we further investigated the direction of effect in a bidirectional MR analysis. In this case, both the effect of the exposure genetic variants on the outcome and the outcome genetic variants to exposure are assessed to inform about the direction of an effect that 
could be cross-generational. We identified independent genetic variants for the relevant outcomes (childhood obesity $\left(p<1 \times 10^{-6}\right)(16)$ and CAD $\left.\left(p<5 \times 10^{-8}\right)(17)\right)$ from their respective GWAS summary statistics and used the steps described before to perform a two-sample MR.

\section{Multivariable MR}

Two Multivariable MR (MVMR) analysis were conducted to assess the effects of genetic liability for ADHD together with genetic liability for childhood obesity or lifetime smoking heaviness on CAD (32). MVMR is an extension of MR that can be used to estimate the causal effects of multiple exposures on one outcome simultaneously (Figure 2). MVMR is explained in more detail elsewhere (33). 


\section{RESULTS}

\section{Estimates of causal effects of ADHD on physical health outcomes}

\section{Cardiometabolic disease and risk factors}

Instrument strength for IVW-MR was assessed using F-statistics for the SNP-exposure association (Table S4) and indicated good instrument strength. MR results are shown in Table 1. There was evidence of a causal effect of genetic liability for ADHD on childhood obesity (OR:1.29 (95\% Cl:1.02,1.63)) and CAD (OR:1.11 (95\% Cl:1.03,1.19)) using IVW. Both weighted median and MR Egger showed effects consistently in the same direction as IVW although with wider confidence intervals (Figure 3). There was little evidence of horizontal pleiotropic effects (MR Egger intercept for childhood obesity: 1.02 (95\% Cl:0.92,1.12); for coronary artery disease: 1.00 (95\% Cl:0.97,1.03)) (Table S5) or heterogeneity between instruments as assessed by Cochrane's Q statistics (Table S4).

There was little evidence of a causal effect of ADHD genetic liability on other cardiometabolic diseases or risk factors. However, instrument heterogeneity (assessed by Cochrane's $Q$ statistic) was detected for BMI $(p=0.006)$, hypertension $\left(p=8 \times 10^{-5}\right)$ and blood pressure $\left(p=8 \times 10^{-5}\right)$, which might undermine the ability to detect a causal relationship.

\section{Neurological diseases}

There was little evidence of a causal effect of ADHD genetic liability on migraine (OR:0.94 (95\% Cl:0.84,1.05)) or epilepsy (OR:1.01 (95\% Cl:1.00,1.02)).

\section{Immunological diseases and cancer}

There was little evidence of a causal effect of ADHD genetic liability for immunological diseases and lung cancer from IVW and weighted median estimates. However, horizontal pleiotropy was detected for inflammatory bowel disease (IBD) (MR Egger intercept :0.94 $(95 \% \mathrm{Cl}: 0.89,0.99))$ and there was evidence of a causal effect from MR Egger (OR:1.93 
$(95 \% \mathrm{Cl}: 1.08,3.45))$. There was little evidence of heterogeneity between instruments, except for allergic rhinitis.

Based on these initial findings, there is some evidence of a causal effect of genetic liability for ADHD on childhood obesity, CAD and IBD. Hence, these three disorders were taken forward for further sensitivity analysis.

(Table 1 here)

\section{Heterogeneity and violation of the NOME assumption}

$\mathrm{I}_{\mathrm{GX}}^{2}$ estimates for childhood obesity $\left(\mathrm{I}_{\mathrm{GX}}^{2}=47 \%\right), \mathrm{CAD}\left(\mathrm{I}_{\mathrm{GX}}{ }_{\mathrm{GX}}=48 \%\right)$ and IBD $\left(\mathrm{I}_{\mathrm{GX}}^{2}=48 \%\right)$ (see supplementary Table $\mathrm{S} 4$ for all $\mathrm{I}_{\mathrm{GX}}^{2}$ estimates) indicate a potential violation of the NOME assumption which might have induced regression dilution bias in MR-Egger estimates. Hence, we performed MR-SIMEX to adjust MR-Egger estimates accordingly (Table S6). The SIMEX point estimate for CAD was consistent with the original IVW and MR Egger estimate (OR:1.11 (95\% Cl:1.03,1.20)). For childhood obesity the MR SIMEX estimate (OR:1.26 (95\% $\mathrm{Cl}: 0.99,1.60)$ ) was increased in comparison to the MR Egger estimate and was closer to the IVW estimate of 1.29. The effect of genetic liability for ADHD on IBD in MR Egger regression was completely attenuated with OR of 1.00 (95\% Cl:0.86,1.17) after SIMEX adjustment, which is in line with the IVW estimate of 0.99 and hence was not taken forward for further analysis.

\section{Bidirectional MR for coronary artery disease and childhood obesity}

To further investigate the direction of observed effects, we performed bidirectional MR to examine the effect of genetic liability for CAD and childhood obesity on ADHD (Table 2). There was little evidence of a causal effect of genetic liability for CAD on ADHD with an IVW estimate of $0.98(95 \% \mathrm{Cl}: 0.92,1.04)$. However, there was some evidence of a causal association of genetic liability for childhood obesity on ADHD with IVW OR=1.15 (95\% $\mathrm{Cl}: 1.05,1.25)$ and weighted median $\mathrm{OR}=1.10$ (95\% Cl:1.01,1.20). MR Egger estimates were 
directionally consistent and there was little evidence of heterogeneity or horizontal pleiotropy (MR Egger intercept: $1.00(95 \% \mathrm{Cl}: 0.88,1.13))$.

(Table 2 here)

\section{Exploratory analyses: Estimates of a causal effect of ADHD on metabolic markers}

The two-sample MR of ADHD on metabolic markers suggested little evidence for a causal effect on any of these markers. (Table S7).

\section{Multivariable MR to test for a potential mediating effect of childhood obesity and lifetime smoking}

Obesity and smoking are established risk factors for CAD (34) that are strongly associated with ADHD (35)'(36) and hence may be mediating the association between the genetic liability for ADHD and CAD. When genetic variants for ADHD and childhood obesity were simultaneously entered in the MVMR model, the direct causal effect of the genetic liability for ADHD on CAD was attenuated to $1.06(95 \% \mathrm{Cl}: 0.95,1.17)$, whereas the effect of the genetic liability for childhood obesity on CAD remained stable (OR:1.14(95\% Cl:1.08,1.20)) (Table S8), which supports a mediating role of childhood obesity. The causal effect of genetic liability for ADHD on lifetime smoking heaviness was 1.07 (95\% Cl:1.04,1.10). When genetic variants for $A D H D$ and lifetime smoking heaviness were simultaneously entered in the MVMR model, the direct causal effect of the genetic liability for ADHD on CAD remained stable (OR:1.10 (95\% Cl:1.00,1.21), whereas the effect of the genetic liability for lifetime smoking heaviness on CAD was attenuated (OR:1.38 (95\% Cl:0.99,1.92)) (Table S9), which supports an effect of genetic liability for ADHD on CAD independent of genetic liability to lifetime smoking heaviness. 


\section{DISCUSSION}

In this study, we employed a two-sample MR approach to test for causal effects of genetic liability for ADHD on previously implicated physical health outcomes. By using genetic variants to proxy for ADHD, we found evidence consistent with a causal effect of genetic liability for ADHD on coronary artery disease $(C A D)$ and evidence for a bidirectional association between genetic liability for ADHD and childhood obesity. There was little evidence for a causal effect on neurological and immunological diseases. The causal effect of genetic liability for ADHD on CAD appears to be (at least partially) mediated by childhood obesity and independent of lifetime smoking.

As reported previously ADHD and genetic liability for ADHD are associated with unhealthy lifestyle and risk behaviours in observational studies (12, 37-39). Patients with ADHD are more likely to smoke $(35)$, be overweight $(36,40)$ and lead a sedentary life (39). All these ADHD outcomes are also risk factors for CAD (34) and hence may act additively as mediators of the observed effect of genetic liability for ADHD on CAD. There are some reports of observational associations between ADHD and cardio-vascular diseases $(41,42)$, although such studies are difficult to conduct due to the latency between exposure and outcome. Here we report evidence supporting a causal effect of genetic liability for ADHD on CAD but not on other cardiovascular risks (such as hypertension). This effect was attenuated when simultaneously assessing the effect of genetic liability for ADHD and childhood obesity on CAD, suggesting childhood obesity as a potential mediator. However, it remained unchanged when adding genetic liability for lifetime smoking, suggesting an effect of genetic liability for ADHD on CAD independent of genetic liability for lifetime smoking heaviness.

Many studies have shown associations between ADHD and obesity in adolescents and adulthood $(36,40,43)$ but the causality of the association has not been established. Hyperactivity is a hallmark of ADHD and it may therefore appear counterintuitive that 
patients with ADHD have a higher risk for obesity (44). However, observational studies have shown that those with ADHD have been reported to spend more time watching television (45), suffer more school exclusions, show lower levels of physical activity, poorer motor skills and increased dysregulation of eating behaviour (44). Our bidirectional MR between genetic liability for ADHD and genetic liability for childhood obesity supports an explanation of shared familial risks including genetic risk in the form of pleiotropic genetic variants that are causal for both ADHD and childhood obesity; and shared environmental/ foetal risk factors among ADHD and childhood obesity (43).

Our results further suggest little evidence supporting a causal role of genetic liability for ADHD on neurological and immunological diseases. One explanation for this might be that there is no causal effect and associations found in observational studies were better explained by other factors, such as confounding. For example, socio-economic factors can confound the observational associations between ADHD and later health outcomes as both ADHD and health behaviours are strongly associated with lower socio-economic status, educational attainment and lower income. Another explanation is that these null-findings might have arisen due to some of the study limitations, such as instrument validity and population stratification, which are discussed in more detail below.

\section{Limitations}

Since the latest ADHD GWAS was made available in November 2017, there are genetic variants that are strongly associated with ADHD but still only explain little variation in the ADHD phenotype (12). The low $I_{G X}^{2}$ estimates suggest substantial heterogeneity in MR Egger regression which is a marker of measurement error in the instruments. This indicates that there might not have been enough power to detect causal associations, since weak instruments bias associations towards the null in two-sample MR studies (27). Furthermore, although there was little statistical evidence for horizontal pleiotropy, the underlying biological pathways leading to ADHD are unknown for most of the genetic variants and 
therefore the possibility of pleiotropic effects of these variants and hence violation of the exclusion restriction assumption cannot be discounted. Since this analysis employed a twosample MR framework, which is based on publicly available data, we were not able to test whether the genetic variants used as instruments are independent of potential confounders of the observed exposure to outcome associations. Confounding may also arise due to population stratification (GWAS sample not representative of the underlying population or GWAS samples of mixed populations)(46), assortative mating (traits not inherited independently and consequent violation of the MR assumption that genetic variants are allocated randomly at conception)(47) or selection bias in the GWAS used, all of which might affect both positive and negative findings of our analyses. Furthermore, estimated twosample MR odds ratios (OR) for associations of binary exposures with binary outcomes can be biased and should only be interpreted in terms of direction and strength of association.

Although MR can overcome some limitations of observational studies, it also makes strong assumptions that cannot always be tested. We therefore highly encourage triangulation of evidence using different causally informative study designs, such as negative control and twin studies (49).

\section{Conclusion}

We found evidence in favour of a causal effect of genetic liability for ADHD on CAD that is potentially mediated by childhood obesity. This strengthens the argument for early treatment and support for children with ADHD and their families and especially promoting physical activity and providing them with dietary advice and support to reduce the future risk for developing obesity and CAD. 


\section{ACKNOWLEGDEMENTS}

We are thankful to the PGC, iPSYCH, GIANT, EGG, CARDloGRAMplusC4D, ILAE, IIBDGC, GABRIEL and ILCCO consortia for making their GWAS summary stats available.

Furthermore, we would like to thank Okada et al., Wootton et al., Scott et al. and the Neale Lab to make their GWAS summary stats available.

\section{FUNDING}

This work was supported by a research grant awarded to AT, GDS, ES and KT by the Wellcome Trust (grant ref: $204895 / Z / 16 / Z)$ supporting BL and LR. CD is funded by the Wellcome Trust (grant ref: 108902/B/15/Z). BL, JRS, GDS, KT and ES work in a unit that receives funding from the University of Bristol and the UK Medical Research Council (MC_UU_00011/1 and MC_UU_00011/3). The funding organisations had no role in the design and conduct of the study; collection, management, analysis and interpretation of the data; preparation, review, or approval of the manuscript; and decision to submit the manuscript for publication

\section{CONTRIBUTIONS}

Dr Beate Leppert had full access to all the data in the study and take responsibility for the integrity of the data and the accuracy of the data analysis.

Concept and design: Leppert, Ajay Thapar, Anita Thapar, Tilling, Davey Smith, Stergiakouli Acquisition, analysis, or interpretation of data: Leppert, Anita Thapar, Tilling, Davey Smith, Stergiakouli

Drafting of the manuscript: Leppert

Critical revision of the manuscript and important intellectual content: Riglin, Staley, Dardani, Anita Thapar, Ajay Thapar, Tilling, Davey Smith

Statistical analysis: Leppert, Dardani, Staley 
bioRxiv preprint doi: https://doi.org/10.1101/630467; this version posted May 10,2019. The copyright holder for this preprint (which was not certified by peer review) is the author/funder, who has granted bioRxiv a license to display the preprint in perpetuity. It is made available under aCC-BY-NC-ND 4.0 International license.

Obtained funding: Thapar, Stergiakouli, Tilling, Davey Smith

Administrative, technical, or material support: Dardani, Staley

Supervision: Anita Thapar, Tilling, Davey Smith, Stergiakouli

\section{CONFLICT OF INTEREST}

No conflict of interest reported. 


\section{REFERENCES}

1. Simon V, Czobor P, Bálint S, Mészáros Á, Bitter I. Prevalence and correlates of adult attention-deficit hyperactivity disorder: meta-analysis. Br J Psychiatry. 2018;194(3):204-11.

2. $\quad$ Fayyad J, De Graaf R, Kessler R, Alonso J, Angermeyer M, Demyttenaere K, et al. Crossnational prevalence and correlates of adult attention-deficit hyperactivity disorder. Br J Psychiatry. 2018;190(5):402-9.

3. Faraone SV, Biederman J, Mick E. The age-dependent decline of attention deficit hyperactivity disorder: A meta-analysis of follow-up studies. Psychol Med. 2006;36(2):159-65.

4. Dalsgaard S, Østergaard SD, Leckman JF, Mortensen PB, Pedersen MG. Mortality in children, adolescents, and adults with attention deficit hyperactivity disorder: a nationwide cohort study. The Lancet. 2015;385(9983):2190-6.

5. Chen Q, Hartman CA, Haavik J, Harro J, Klungsoyr K, Hegvik TA, et al. Common psychiatric and metabolic comorbidity of adult attention-deficit/hyperactivity disorder: A population-based cross-sectional study. PLoS One. 2018;13(9):e0204516.

6. Instanes JT, Klungsoyr K, Halmoy A, Fasmer OB, Haavik J. Adult ADHD and Comorbid Somatic Disease: A Systematic Literature Review. J Atten Disord. 2018;22(3):203-28.

7. Tylee DS, Sun J, Hess JL, Tahir MA, Sharma E, Malik R, et al. Genetic correlations among psychiatric and immune-related phenotypes based on genome-wide association data. Am J Med Genet B Neuropsychiatr Genet. 2018;177(7):641-57.

8. Alabaf S, Gillberg C, Lundstrom S, Lichtenstein P, Kerekes N, Rastam M, et al. Physical health in children with neurodevelopmental disorders. J Autism Dev Disord. 2018; epub ahead of print 24 July 2018(10.1007/s10803-018-3697-4).

9. Doshi JA, Hodgkins P, Kahle J, Sikirica V, Cangelosi MJ, Setyawan J, et al. Economic impact of childhood and adult attention-deficit/hyperactivity disorder in the United States. J Am Acad Child Adolesc Psychiatry. 2012;51(10):990-1002.

10. Parsonage M. The lifetime costs of attention deficit hyperactivity disorder (ADHD). Centre for Mental Health. 2014.

11. Davey Smith G, Ebrahim S. Epidemiology--is it time to call it a day? Int J Epidemiol. 2001;30(1):1-11.

12. Demontis D, Walters RK, Martin J, Mattheisen M, Als TD, Agerbo E, et al. Discovery of the first genome-wide significant risk loci for attention deficit/hyperactivity disorder. Nat Genet. 2019;51(1):63-75.

13. Davey Smith G, Ebrahim S. 'Mendelian randomization': can genetic epidemiology contribute to understanding environmental determinants of disease? Int J Epidemiol. 2003;32(1):1-22.

14. Davies NM, Holmes MV, Davey Smith G. Reading Mendelian randomisation studies: a guide, glossary, and checklist for clinicians. BMJ. 2018;362:k601.

15. Locke AE, Kahali B, Berndt SI, Justice AE, Pers TH, Day FR, et al. Genetic studies of body mass index yield new insights for obesity biology. Nature. 2015;518:197.

16. Bradfield JP, Taal HR, Timpson NJ, Scherag A, Lecoeur C, Warrington NM, et al. A genomewide association meta-analysis identifies new childhood obesity loci. Nat Genet. 2012;44(5):526-31.

17. Nikpay M, Goel A, Won HH, Hall LM, Willenborg C, Kanoni S, et al. A comprehensive 1,000 Genomes-based genome-wide association meta-analysis of coronary artery disease. Nat Genet. 2015;47(10):1121-30.

18. Churchhouse C, Neale B. RAPID GWAS OF THOUSANDS OF PHENOTYPES FOR 337,000 SAMPLES IN THE UK BIOBANK 2017 [updated 20.09.2017. Available from: http://www.nealelab.is/blog/2017/7/19/rapid-gwas-of-thousands-of-phenotypes-for-337000samples-in-the-uk-biobank.

19. Scott RA, Scott L, Magi R, Marullo L, Gaulton KJ, Kaakinen M, et al. An Expanded GenomeWide Association Study of Type 2 Diabetes in Europeans. Diabetes. 2017;66(11):2888-902. 
20. International League Against Epilepsy Consortium on Complex E. Genome-wide megaanalysis identifies 16 loci and highlights diverse biological mechanisms in the common epilepsies. Nat Commun. 2018;9(1):5269.

21. Okada Y, Wu D, Trynka G, Raj T, Terao C, Ikari K, et al. Genetics of rheumatoid arthritis contributes to biology and drug discovery. Nature. 2014;506(7488):376-81.

22. Liu JZ, van Sommeren S, Huang H, Ng SC, Alberts R, Takahashi A, et al. Association analyses identify 38 susceptibility loci for inflammatory bowel disease and highlight shared genetic risk across populations. Nat Genet. 2015;47(9):979-86.

23. Moffatt MF, Gut IG, Demenais F, Strachan DP, Bouzigon E, Heath S, et al. A large-scale, consortium-based genomewide association study of asthma. N Engl J Med. 2010;363(13):1211-21.

24. Consortium TaGLEE, Paternoster L, Standl M, Waage J, Baurecht H, Hotze M, et al. Multiancestry genome-wide association study of 21,000 cases and 95,000 controls identifies new risk loci for atopic dermatitis. Nat Genet. 2015;47:1449.

25. Wang Y, McKay JD, Rafnar T, Wang Z, Timofeeva MN, Broderick $P$, et al. Rare variants of large effect in BRCA2 and CHEK2 affect risk of lung cancer. Nat Genet. 2014;46(7):736-41.

26. Kettunen J, Demirkan A, Wurtz P, Draisma HH, Haller T, Rawal R, et al. Genome-wide study for circulating metabolites identifies 62 loci and reveals novel systemic effects of LPA. Nat Commun. 2016;7:11122.

27. Pierce BL, Burgess S. Efficient design for Mendelian randomization studies: subsample and 2sample instrumental variable estimators. Am J Epidemiol. 2013;178(7):1177-84.

28. Lawlor DA, Harbord RM, Sterne JA, Timpson N, Davey Smith G. Mendelian randomization: using genes as instruments for making causal inferences in epidemiology. Stat Med. 2008;27(8):1133-63.

29. Bowden J, Davey Smith G, Haycock PC, Burgess S. Consistent Estimation in Mendelian Randomization with Some Invalid Instruments Using a Weighted Median Estimator. Genet Epidemiol. 2016;40(4):304-14.

30. Bowden J, Davey Smith G, Burgess S. Mendelian randomization with invalid instruments: effect estimation and bias detection through Egger regression. Int J Epidemiol. 2015;44(2):512-25.

31. Bowden J, Del Greco MF, Minelli C, Davey Smith G, Sheehan NA, Thompson JR. Assessing the suitability of summary data for two-sample Mendelian randomization analyses using MR-Egger regression: the role of the 12 statistic. Int J Epidemiol. 2016;45(6):1961-74.

32. Wootton RE, Richmond RC, Stuijfzand BG, Lawn RB, Sallis HM, Taylor GMJ, et al. Causal effects of lifetime smoking on risk for depression and schizophrenia: Evidence from a Mendelian randomisation study. bioRxiv. 2018:381301.

33. Sanderson E, Davey Smith G, Windmeijer F, Bowden J. An examination of multivariable Mendelian randomization in the single-sample and two-sample summary data settings. Int $J$ Epidemiol. 2018:dyy262-dyy.

34. Khot UN, Khot MB, Bajzer CT, et al. Prevalence of conventional risk factors in patients with coronary heart disease. JAMA. 2003;290(7):898-904.

35. Anttila V, Bulik-Sullivan B, Finucane HK, Walters RK, Bras J, Duncan L, et al. Analysis of shared heritability in common disorders of the brain. Science (New York, NY). 2018;360(6395).

36. Nigg JT, Johnstone JM, Musser ED, Long HG, Willoughby MT, Shannon J. Attentiondeficit/hyperactivity disorder (ADHD) and being overweight/obesity: New data and meta-analysis. Clin Psychol Rev. 2016;43:67-79.

37. Franke B, Michelini G, Asherson P, Banaschewski T, Bilbow A, Buitelaar JK, et al. Live fast, die young? A review on the developmental trajectories of ADHD across the lifespan. Eur Neuropsychopharmacol. 2018;28(10):1059-88.

38. Davis C. Attention-deficit/hyperactivity disorder: associations with overeating and obesity. Curr Psychiatry Rep. 2010;12(5):389-95. 
39. Quesada D, Ahmed NU, Fennie KP, Gollub EL, Ibrahimou B. A Review: Associations Between Attention-deficit/hyperactivity Disorder, Physical Activity, Medication Use, Eating Behaviors and Obesity in Children and Adolescents. Arch Psychiatr Nurs. 2018;32(3):495-504.

40. Cortese S, Tessari L. Attention-Deficit/Hyperactivity Disorder (ADHD) and Obesity: Update 2016. Curr Psychiatry Rep. 2017;19(1):4.

41. Wei YJ, Zhu Y, Liu W, Bussing R, Winterstein AG. Prevalence of and Factors Associated With Long-term Concurrent Use of Stimulants and Opioids Among Adults With AttentionDeficit/Hyperactivity Disorder. JAMA. 2018;1(4):e181152.

42. Maher GM, O'Keeffe GW, Kearney PM, et al. Association of hypertensive disorders of pregnancy with risk of neurodevelopmental disorders in offspring: A systematic review and metaanalysis. JAMA Psychiatry. 2018;75(8):809-19.

43. Hanc T, Cortese S. Attention deficit/hyperactivity-disorder and obesity: A review and model of current hypotheses explaining their comorbidity. Neurosci Biobehav Rev. 2018;92:16-28.

44. Khalife N, Kantomaa M, Glover V, Tammelin T, Laitinen J, Ebeling $\mathrm{H}$, et al. Childhood attention-deficit/hyperactivity disorder symptoms are risk factors for obesity and physical inactivity in adolescence. J Am Acad Child Adolesc Psychiatry. 2014;53(4):425-36.

45. Lingineni RK, Biswas S, Ahmad N, Jackson BE, Bae S, Singh KP. Factors associated with attention deficit/hyperactivity disorder among US children: results from a national survey. BMC pediatrics. 2012;12:50.

46. Munafò $M$, Davey Smith G. Biased estimates in mendelian randomization studies conducted in unrepresentative samples. JAMA Cardiology. 2018;3(2):181-.

47. Hartwig FP, Davies NM, Davey Smith G. Bias in Mendelian randomization due to assortative mating. Genet Epidemiol. 2018;42(7):608-20.

48. Burgess S, Labrecque JA. Mendelian randomization with a binary exposure variable: interpretation and presentation of causal estimates. Eur J Epidemiol. 2018;33(10):947-52.

49. Lawlor DA, Tilling K, Davey Smith G. Triangulation in aetiological epidemiology. Int J Epidemiol. 2016;45(6):1866-86. 


\section{FIGURE LEGENDS}

Figure 1. Flow chart of MR study design.

Figure 2: Hypothetical relationship between genetic variants $(G)$, exposures $X_{1}$ and $\mathrm{X}_{2}$ and an outcome $\mathrm{Y}$ under the presence of unobserved confounders $(\mathrm{U})$.

Figure 3: Effect estimates of single variants for $A D H D$ on childhood obesity $(A+B)$ and coronary artery disease $(C A D)(C+D)$ using IVW, weighted median and MR Egger approaches. Estimates are shown as log odds $\pm S E(A+C)$ and $\log$ odds \pm $95 \% \mathrm{Cl}(\mathrm{B}+\mathrm{D})$. 
TABLES

Table 1: Causal estimates for ADHD on physical diseases using IVW, weighted median and MR Egger regression.

\begin{tabular}{|c|c|c|c|c|c|c|c|}
\hline \multirow{2}{*}{ Disease } & \multirow{2}{*}{ SNPs } & \multicolumn{2}{|l|}{ IVW } & \multicolumn{2}{|c|}{ Weighted median } & \multicolumn{2}{|c|}{ MR Egger } \\
\hline & & $O R(95 \% \mathrm{Cl})$ & $p$-value & $O R(95 \% C l)$ & $p$-value & $O R(95 \% \mathrm{Cl})$ & $p$-value \\
\hline \multicolumn{8}{|c|}{ Cardiometabolic disease and risk factors } \\
\hline BMI & 10 & {$[0.03(-0.01,0.08)]$} & 0.166 & {$[0.00(-0.05,0.04)]$} & 0.832 & {$[-0.07(-0.28,0.14)]$} & 0.541 \\
\hline Childhood obesity & 9 & $1.29(1.02,1.63)$ & 0.033 & $1.13(0.82,1.56)$ & 0.444 & $1.06(0.37,3.07)$ & 0.919 \\
\hline Coronary artery disease & 11 & $1.11(1.03,1.19)$ & 0.006 & $1.09(0.99,1.20)$ & 0.067 & $1.11(0.78,1.57)$ & 0.584 \\
\hline Myocardial infarction & 11 & $1.06(0.97,1.16)$ & 0.191 & $1.05(0.94,1.16)$ & 0.424 & $1.11(0.71,1.73)$ & 0.654 \\
\hline Hypertension & 11 & $1.05(0.97,1.13)$ & 0.252 & $1.04(0.97,1.11)$ & 0.247 & $0.95(0.66,1.37)$ & 0.793 \\
\hline Systolic blood pressure & 11 & {$[-0.01(-0.05,0.03)]$} & 0.550 & {$[-0.01(-0.04,0.03)]$} & 0.746 & {$[-0.04(-0.21,0.13)]$} & 0.637 \\
\hline Type II diabetes mellitus & 11 & $1.09(1.00,1.20)$ & 0.058 & $1.11(0.98,1.25)$ & 0.102 & $0.92(0.61,1.37)$ & 0.677 \\
\hline \multicolumn{8}{|l|}{ Neurological diseases } \\
\hline Migraine & 12 & $0.94(0.84,1.05)$ & 0.268 & $0.89(0.76,1.03)$ & 0.111 & $0.64(0.40,1.04)$ & 0.099 \\
\hline Epilepsy & 9 & $1.01(1.00,1.02)$ & 0.139 & $1.01(0.99,1,03)$ & 0.181 & $1.00(0.95,1.07)$ & 0.906 \\
\hline \multicolumn{8}{|l|}{ Immunological diseases } \\
\hline Rheumatoid arthritis & 10 & $1.02(0.87,1.19)$ & 0.823 & $1.10(0.88,1.36)$ & 0.405 & $1.00(0.47,2.12)$ & 0.997 \\
\hline Inflammatory bowel disease & 11 & $0.99(0.86,1.15)$ & 0.925 & $0.96(0.81,1.14)$ & 0.654 & $1.93(1.08,3.45)$ & 0.052 \\
\hline Allergic rhinitis & 11 & $0.92(0.80,1.07)$ & 0.281 & $0.86(0.75,0.99)$ & 0.038 & $1.10(0.57,2.13)$ & 0.783 \\
\hline Asthma & 8 & $1.16(0.93,1.45)$ & 0.191 & $1.13(0.88,1.46)$ & 0.331 & $0.64(0.14,2.97)$ & 0.591 \\
\hline Eczema & 11 & $0.94(0.82,1.07)$ & 0.339 & $0.98(0.82,1.1)$ & 0.835 & $0.79(0.43,1.46)$ & 0.474 \\
\hline
\end{tabular}

\section{Cancer}

Lung Cancer

10

$1.10(0.96,1.27)$

0.168

1.08(0.89,1.31)

0.436

$1.04(0.56,1.94)$

0.899

Odds ratios for binary outcomes are to be interpreted as a change in the log odds ratio of the outcome per unit increase in the log odds ratio of ADHD

[ ] estimates are presented as beta $(95 \% \mathrm{Cl})$ 
Table 2: Bidirectional MR with causal estimates for childhood obesity, coronary artery disease and inflammatory bowel disease on ADHD using IVW, weighted median and MR Egger regression.

\begin{tabular}{|c|c|c|c|c|c|c|c|}
\hline \multirow{2}{*}{ Disease } & \multirow{2}{*}{ SNPs } & \multicolumn{2}{|l|}{ IVW } & \multicolumn{2}{|c|}{ Weighted median } & \multicolumn{2}{|c|}{ MR Egger } \\
\hline & & $O R(95 \% \mathrm{Cl})$ & $p$-value & OR(95\%Cl) & $p$-value & $O R(95 \% C l)$ & $p$-value \\
\hline Childhood obesity & 7 & $1.15(1.05,1.25)$ & 0.003 & $1.10(1.01,1.20)$ & 0.031 & $1.16(0.60,2.25)$ & 0.677 \\
\hline Coronary artery disease & 37 & $0.98(0.92,1.04)$ & 0.468 & $0.96(0.88,1.05)$ & 0.346 & $0.91(0.79,1.06)$ & 0.224 \\
\hline
\end{tabular}

SNP - Single nucleotide polymorphism, IVW - Inverse variant weighted regression, MR - Mendelian randomization, OR - Odds ratio, CI - Confidence interval

Odds ratios for binary outcomes are to be interpreted as a change in the log odds ratio of the outcome per unit increase in the log odds ratio of the exposure 
bioRxiv preprint doi: https://doi.org/10.1101/630467; this version posted May 10, 2019. The copyright holder for this preprint (which was not certified by peer review) is the author/funder, who has granted bioRxiv a license to display the preprint in perpetuity. It is made available under aCC-BY-NC-ND 4.0 International license.

\section{Two-sample MR}

Genetic variants for exposure and outcome are available from independent GWAS drawn from the same population

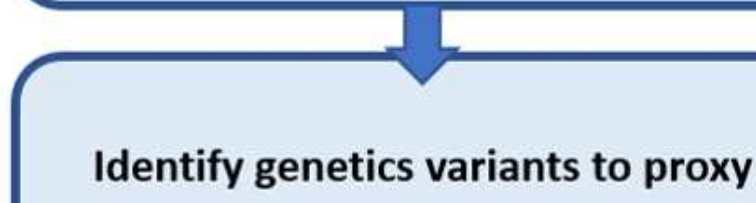
exposure

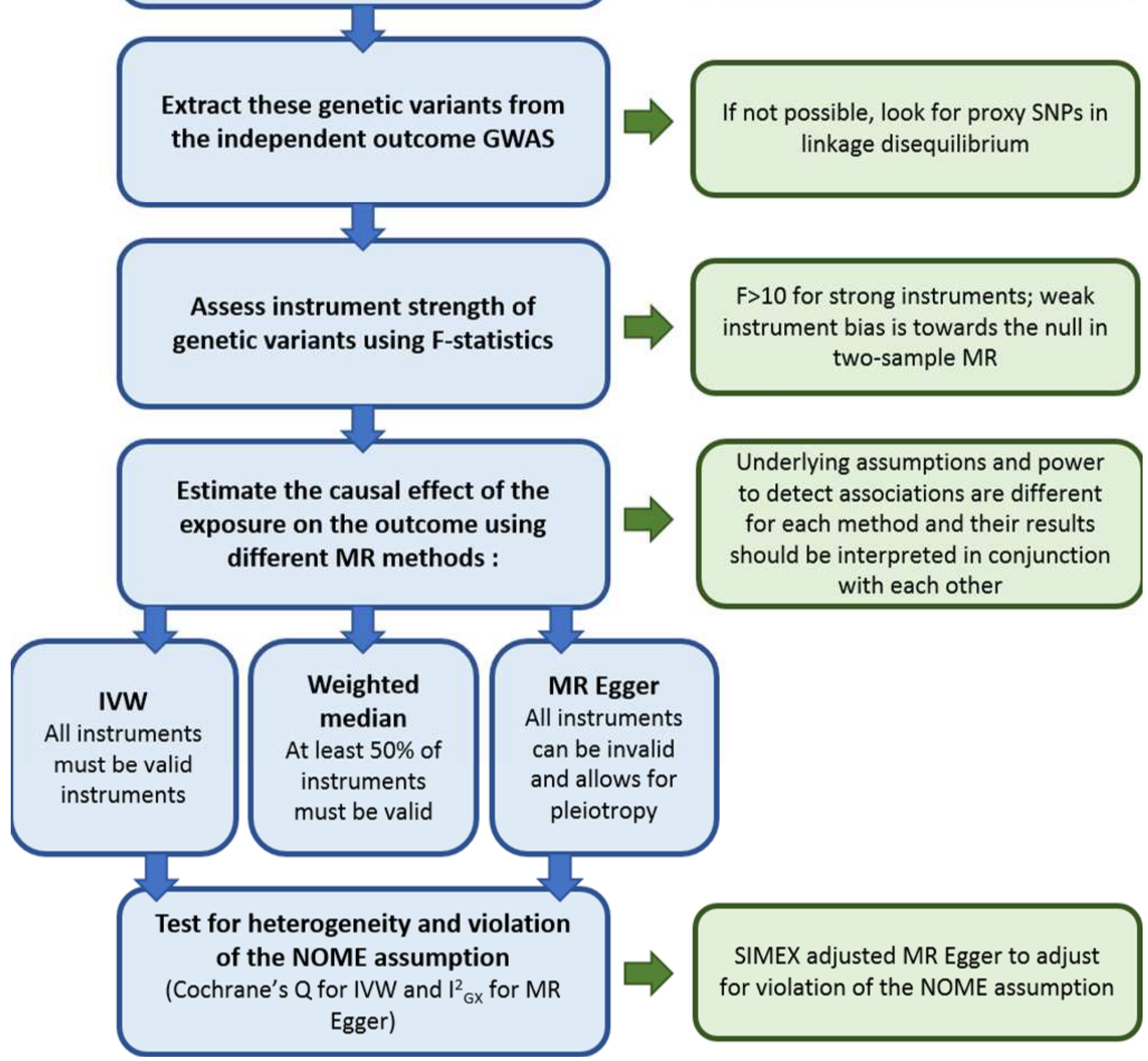

One or more independent SNPS identified by well-powered GWAS

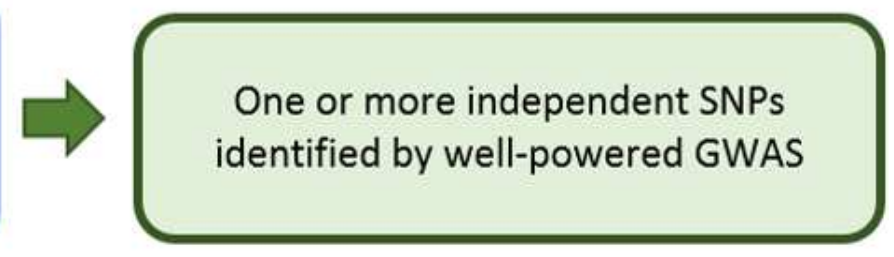




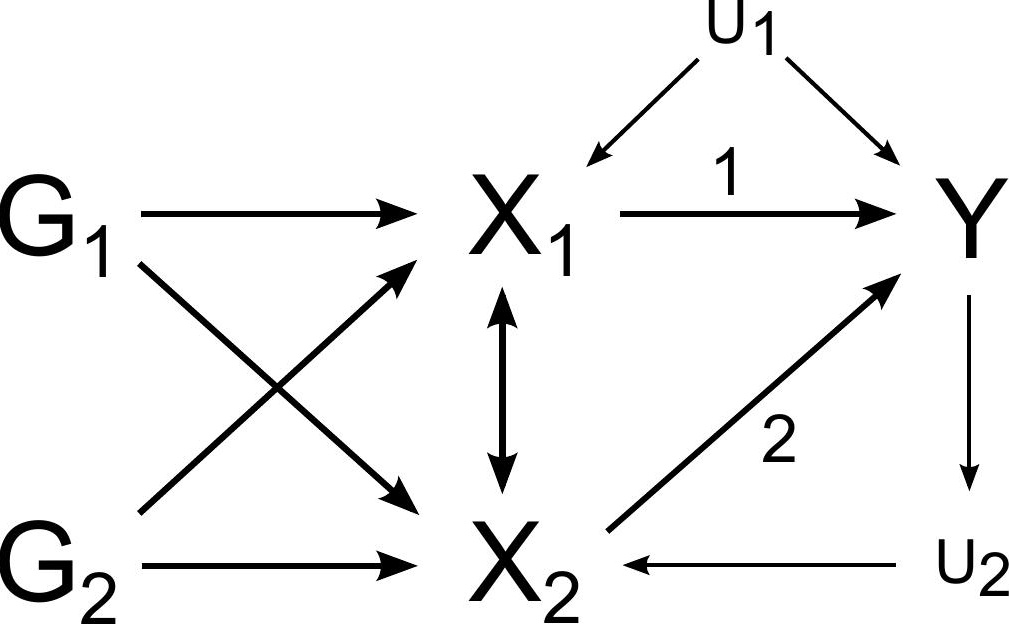




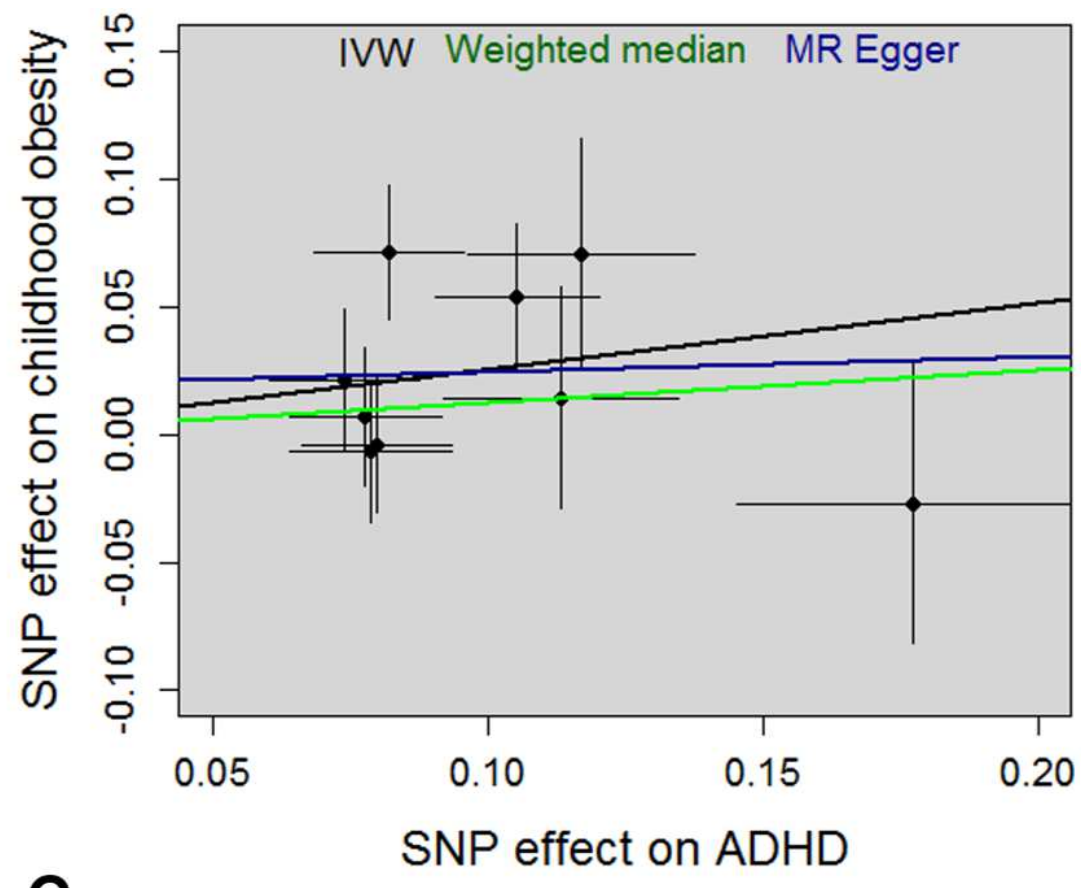

rs1427829

rs212178

rs17531412

rs10262192

rs9677504

rs4916723

rs 8039398

rs227378

rs118041269

All - Inverse variance weighted

All - Weighted median

All - MR Egger
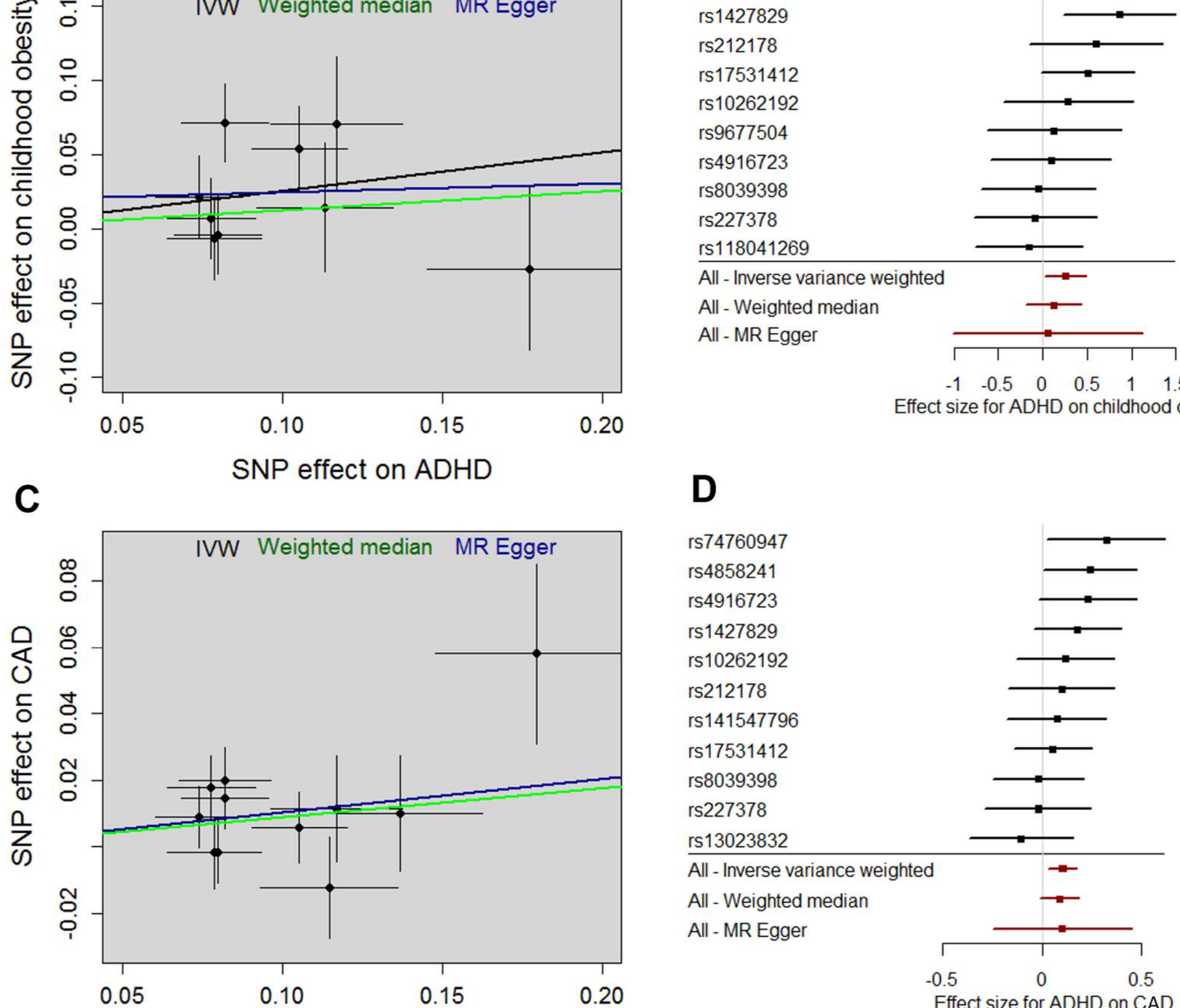

rs74760947

rs 4858241

rs4916723

rs1427829

rs10262192

rs212178

rs141547796

rs 17531412

rs8039398

rs227378

rs13023832

All - Inverse variance weighted

All - Weighted median

All - MR Egger

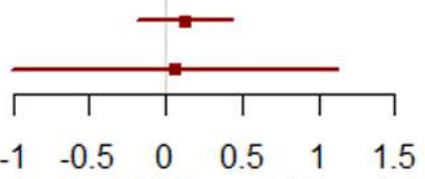

Effect size for ADHD on childhood obesity

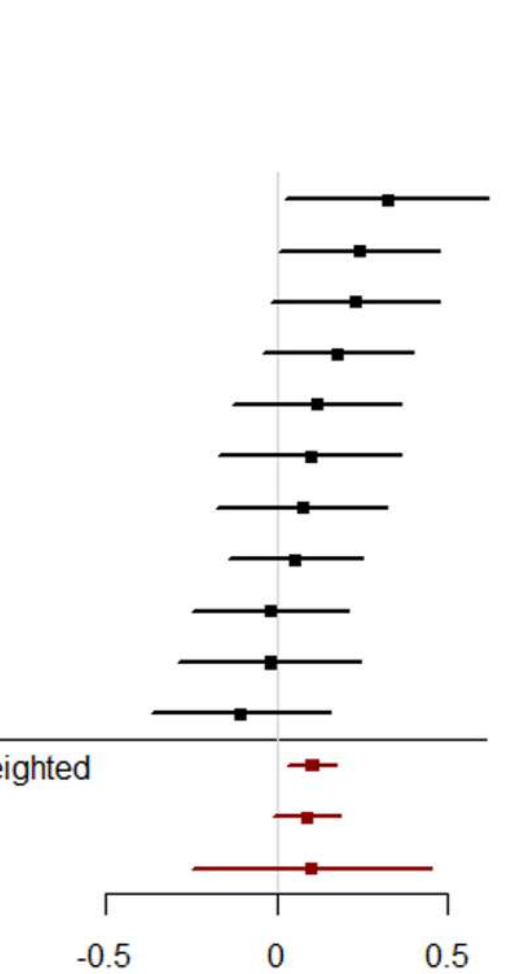

Effect size for ADHD on CAD 